\title{
INTEGRATION OF THE NORMAL POWER APPROXIMATION
}

\author{
GotTFried Berger \\ Stamford, U.S.A.
}

I. Consider the set of functions

$$
\pi_{j}(x)=\int_{x}^{\infty}(t-x)^{j} d F(t), j=0, \mathrm{I}, \ldots
$$

Obviously, $\pi_{1}(x)$ represents the net premium of the excess cover over the priority $x$, and $\sigma^{2}(x)=\pi_{2}(x)-\pi_{1}^{2}(x)$ the variance thereof.

If a distribution function $F(x)=\mathbf{I}-\pi_{0}(x)$ is given, the set (I) can be generated by means of the recursion formulae

$$
\pi_{j}^{\prime}(x)=-j \pi_{j-1}(x), j=\mathrm{I}, 2, \ldots
$$

2. Let us study the special class of d.fs. $F(x)$ which satisfy

$$
F(x)=\Phi(y) \equiv \frac{\mathrm{I}}{\sqrt{2 \pi}} \int_{-\infty}^{y} e^{-1 / 2 t^{2}} d t,
$$

where

$$
x=\Delta(y) \equiv \beta_{0}+\beta_{1} y+\ldots+\beta_{k} y^{k} .
$$

If these conditions are met, the integrals (I) have the solution:

$$
\pi_{j}(x)=A_{j}(y) \cdot(\mathrm{I}-\Phi(y))+B_{j}(y) \cdot \Phi^{\prime}(y) .
$$

$A_{j}(y)$ and $B_{j}(y)$, respectively, are polynomials of rank $j k$ and $j k-\mathrm{I}$. Their coefficients are determined by the equations:

$$
\begin{aligned}
& A_{j}^{\prime}(y)=-j \Delta^{\prime}(y) \cdot A_{j-1}(y), \\
& B_{j}^{\prime}(y)=-j \Delta^{\prime}(y) \cdot B_{j-1}(y)+A_{j}(y)+y B_{j}(y), \\
& A_{0}(y)=\mathrm{I}, \\
& B_{0}(y)=0 .
\end{aligned}
$$

The system (5) is obtained by differentiation of (4) with respect to $y$, and observing (2). 
3. The idea behind the normal power expansion is to apply (3a) as approximation, subject to a transformation $x=\Delta(y)$. Preferably the parameters of $\Delta(y)$ should not depend on the particular choice of $y$ or $x$, but only on general characteristics of the d.f. $F(x)$, such as $E=\pi_{1}(0), \sigma=\sigma(0), \gamma_{1}=$ skewness and $\gamma_{2}=$ excess.

Kauppi and Ojantakanen [I] have tackled the problem to define functions $x=\Delta(y)$, which make (3a) a reasonable approximation. They found three suitable expressions $\Delta(y)$, one of them-credited to Loimaranta - has the form ( $3 \mathrm{~b})$ and this one became known as the normal power expansion. Under this method (see BeardPentikaeinen-Pesonen [2]) the coefficients $\beta_{i}$ of $(3 \mathrm{~b})$ are determined by reversion of the Edgeworth expansion as follows:

$$
\begin{aligned}
\frac{x-E}{\sigma}=y & +\frac{\gamma_{1}}{6}\left(y^{2}-\mathrm{I}\right) \\
& +\frac{\gamma_{2}}{24}\left(y^{3}-3 y\right)-\left(\frac{\gamma_{1}}{6}\right)^{2}\left(2 y^{3}-5 y\right) \\
& +\ldots
\end{aligned}
$$

We may denote by $\mathrm{NPk}$ the normal power approximation, which uses the first $k$ terms of (6). Then, NPI corresponds to the well known normal approximation.

$\mathrm{NP}_{2}$ uses the first line of (6) only, and $\mathrm{NP}_{3}$ everything which is written out. Thus, $\mathrm{NP}_{3}$ requires the solution of a cubic equation.

4. The methods $\mathrm{NP}_{2}$ and $\mathrm{NP}_{3}$ were programmed in APL. This required about 20 lines, including the subprograms to solve (5), the quadratic or cubic equation (6), and to determine $\Phi(y)$ and $\Phi^{\prime}(y)$.

The cubic equation for $\mathrm{NP}_{3}$ has in some relevant cases 3 real roots. It is necessary therefore to program rules to select the meaningful of several real roots $y$.

On an IBM $37^{\circ}$, the CPU time needed to calculate $\pi_{0}(x), \pi_{1}(x)$ and $\sigma(x)$ for a set of 6 values $x$ was $x$ second for $\mathrm{NP}_{2}$, and 2.4 seconds for $\mathrm{NP}_{3}$.

5. The NP approximations were applied first to a life insurance distribution similar to the one used by Ammeter [3]. The result is 
shown in Table $I$. The exact values were obtained by another APL program, the CPU time needed was:

$$
\begin{aligned}
28 \text { seconds for } t= & \text { I00 } \\
3.2 \text { seconds for } t= & \text { Io } \\
\mathrm{I} \text { seconds for } t= & \mathrm{I}
\end{aligned}
$$

Thus, the approximation technique makes economical sense only, if the number $t$ of expected claims is at least to or more.

As another example, the non-industrial fire distribution from the work of Bohman-Escher [4] was chosen. Table 2 shows a comparison with correct values from [4], Table 3 some additional comparisons with numerical results from Seal [5].

6. The comparisons contained in the Tables $I$ to 3 point out the following suggestions:

a) The integration does not seem to enlarge the error margin. Thus, the NP technique can be applied to estimate stop loss gross premiums.

b) $\mathrm{NP}_{2}$ yields quite reasonable results, if $\gamma_{1} \leq 2$. This corresponds with previous experience.

c) $\mathrm{NP}_{3}$ does not generally produce better results than $\mathrm{NP}_{2}$. It appears that $\mathrm{NP}_{3}$ is preferable only for lower values of $x$ (say $x \leq E+2 \sigma)$.

d) $\mathrm{NP}_{3}$ yields reasonable results even in the Life case with $\gamma_{1}=4.3$, but not in the Fire cases with $\gamma_{1}=3.5$ and 3.8 (not even in the vicinity of $x=E$ ). It may be that not only $\gamma_{1}$, but also the relation $E \gamma_{1} / \sigma$ is a criterion of goodness of fit.

\section{REFERENCES}

[I] Kauppi, OJantakanen (I969): "Approximations of the generalized Poisson function"; Astin Bulletin.

[2] Beard. Pentikaeinen, Pesonen (I969): "Risk Theory"; Methuen, London.

[3] Ammeter (1955). "The calculation of premium rates for excess of loss and stop loss reassurance treaties"; Arithbel, Brussels.

[4] Bohman, Escher (I964): "Studies in Risk Theory..."; Skand. Aktu. Tidskr.

[5] SEAL (I97I): "Numerical calculation of the Bohman-Escher family convolution-mixed negative binomial distribution functions"; MVSM. 


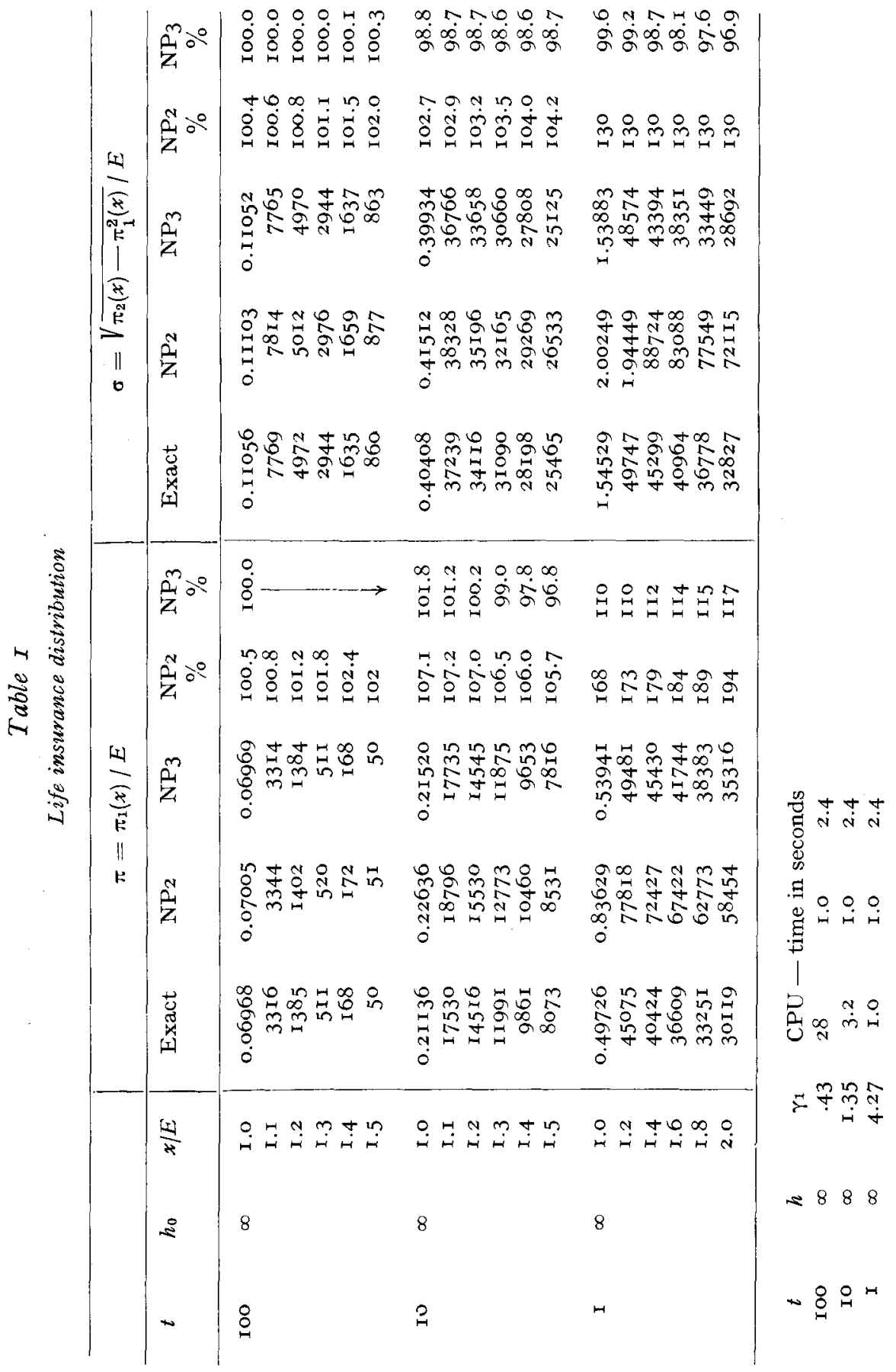




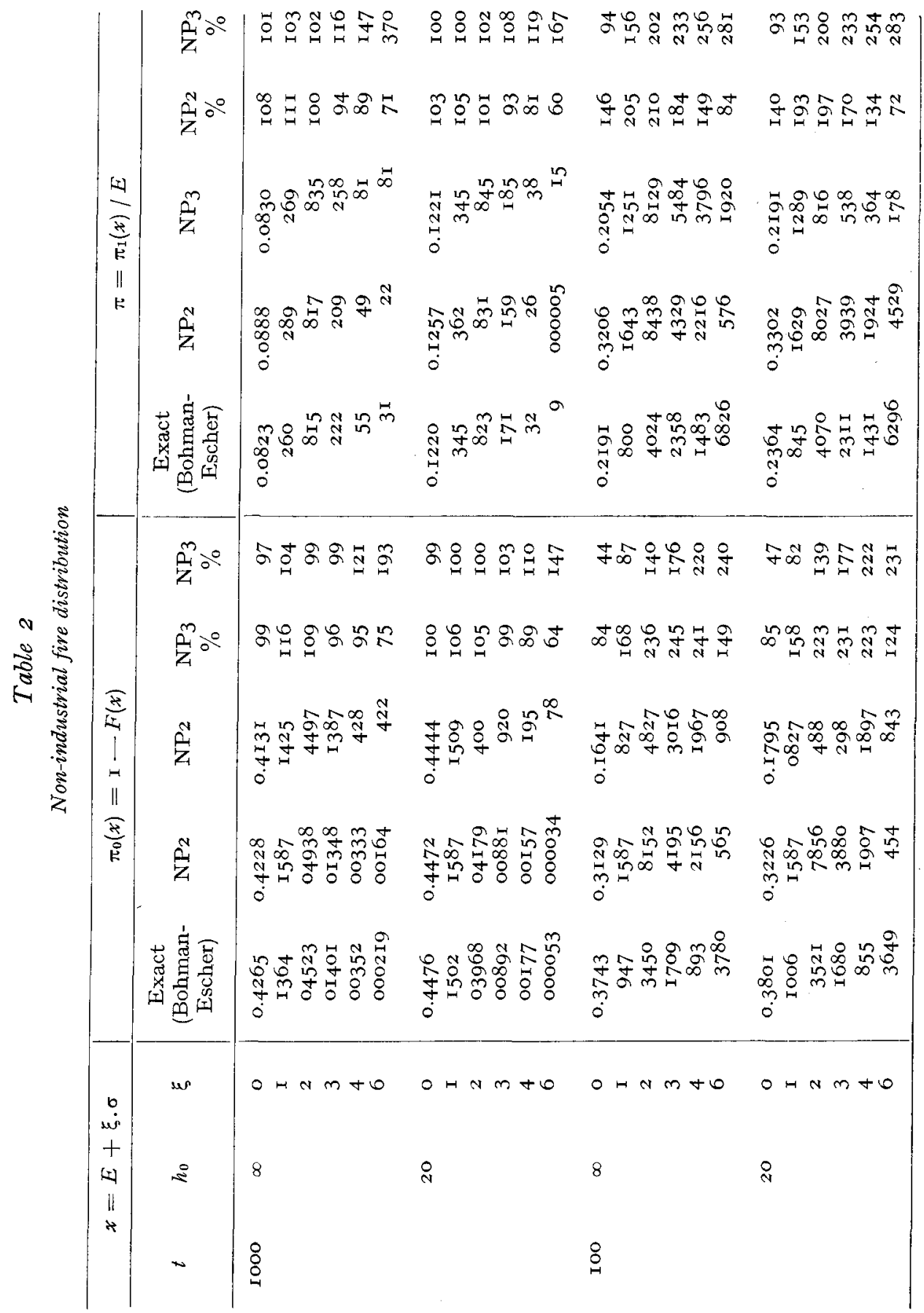


Table 3

Non-industrial fire distribution

\begin{tabular}{|c|c|c|c|c|c|c|c|c|}
\hline \multicolumn{4}{|c|}{$x=E+\xi \cdot \sigma$} & \multicolumn{5}{|c|}{$\pi_{0}(x)=\mathrm{I}-F(x)$} \\
\hline$t$ & $h_{0}$ & $x / E$ & $\xi$ & $\begin{array}{l}\text { Exact } \\
\text { (Seal) }\end{array}$ & $\mathrm{NP}_{2}$ & $\mathrm{NP}_{3}$ & $\begin{array}{c}\mathrm{NP}_{2} \\
\%\end{array}$ & $\underset{\%}{\mathrm{NP}_{3}}$ \\
\hline \multirow[t]{5}{*}{1000} & I & .5 & & $0.5977^{8}$ & 0.5645 & $0.5^{825}$ & 94 & 97 \\
\hline & & I.O & o & 36710 & 3805 & 3593 & IO4 & 98 \\
\hline & & & I & I353I & I 587 & I 347 & I I 7 & 100 \\
\hline & & & 3 & 1839 & 229 & I94 & I 24 & 106 \\
\hline & & & 5 & 250 & 28 & 29 & I 3 & II 7 \\
\hline \multirow[t]{5}{*}{ IOO } & I & .5 & & $0.547^{\circ}$ & 0.4905 & 0.4846 & 90 & 89 \\
\hline & & I.O & o & 3448 & $354^{\circ}$ & 3040 & IO3 & 88 \\
\hline & & & I & 1 226 & I 587 & I I 89 & I 29 & 97 \\
\hline & & & 3 & 198 & 297 & 238 & I 50 & 120 \\
\hline & & & 5 & 46 & $5 \mathrm{I}$ & 56 & III & I 22 \\
\hline
\end{tabular}

The total claim distributions being tested have these statistical measures:

\begin{tabular}{lllll}
\hline$t$ & $h_{0}$ & $\sigma / E$ & $\gamma_{1}$ & $\gamma_{2}$ \\
\hline
\end{tabular}

Life:

$\begin{array}{rrrrr}\text { IOO } & \infty & . \mathrm{I} 75 & .427 & .246 \\ \text { Io } & \infty & .554 & \mathrm{I} .35 \mathrm{I} & 2.459 \\ \mathrm{I} & \infty & \mathrm{I} .75 \mathrm{I} & 4.27 \mathrm{I} & 24.590\end{array}$

Non-industrial Fire:

IOOO

$\begin{array}{rr}\infty & .218 \\ 20 & .312 \\ \mathrm{I} & \mathrm{I} .024\end{array}$

IOO

$\begin{array}{rr}\infty & .690 \\ 20 & .725 \\ \mathrm{I} & \mathrm{I} .2 \mathrm{I} 5\end{array}$

I. 214

2.010

2.624

I. 153

6.045

26.234

22.577

10.729 